\title{
Patients with chronic pain lack somatic markers during decision-making
}

\author{
This article was published in the following Dove Press journal: \\ Journal of Pain Research \\ 15 July 2014 \\ Number of times this article has been viewed
}

\author{
Nicolas-Andreas Elvemo' \\ Kristian Bernhard Nilsen ${ }^{1,2}$ \\ Nils Inge Landrø $\varnothing^{3,4}$ \\ Petter Christian \\ Borchgrevink ${ }^{4,5}$ \\ Asta Kristine Håberg ${ }^{1,6}$ \\ 'Department of Neuroscience, \\ Norwegian University of Science \\ and Technology, Trondheim, Norway; \\ ${ }^{2}$ Department of Neurology, Section \\ for Clinical Neurophysiology, Oslo \\ University Hospital, Oslo, Norway; \\ ${ }^{3}$ Clinical Neuroscience Research \\ Group, Department of Psychology, \\ University of Oslo, Oslo, Norway; \\ ${ }^{4}$ Department of Circulation and \\ Medical Imaging, Norwegian \\ University of Science and Technology, \\ Trondheim, Norway; ${ }^{5}$ Department of \\ Anesthesiology, St Olav University \\ Hospital, Trondheim, Norway; \\ ${ }^{6}$ Department of Medical Imaging, \\ St Olav University Hospital, \\ Trondheim, Norway
}

\begin{abstract}
Patients with chronic pain have impaired cognitive functions, including decision making, as shown with the Iowa gambling task (IGT). The main aim of this study was to elucidate whether patients' decision making is associated with a lack of the anticipatory skin conductance response (SCR). An increase in anticipatory SCR before making unfavorable choices is known to guide decisions in healthy controls during the IGT. Since several brain regions involved in decision making are reported to have altered morphology in patients with chronic pain, the second aim was to explore the associations between IGT performance and brain structure volumes. Eighteen patients with chronic pain of mixed etiology and 19 healthy controls matched in terms of age, sex, and education were investigated with a computerized IGT during the recording of SCR, heart rate, and blood pressure. The participants also underwent neuropsychological testing, and three-dimensional T1-weighted cerebral magnetic resonance images were obtained. Contrary to controls, patients did not generate anticipatory SCRs before making unfavorable choices, and they switched between decks of cards during the late phase of the IGT significantly more often, and this was still observed after adjusting for depression scores. None of the other autonomic measures differed during IGT performance in either group or between groups. In patients, IGT scores correlated positively with total cortical grey matter volume. In controls, there was no such association, but their IGT scores correlated with the anticipatory SCR. It may be speculated that the reduction in anticipatory SCRs makes the chronic pain patients rely more on cortical resources during decision making.
\end{abstract}

Keywords: Iowa gambling task, skin conductance response, autonomic measures, magnetic resonance imaging, cortex

\section{Introduction}

Patients with chronic pain have impaired cognitive functions; ${ }^{1}$ among those are impaired decision making, ${ }^{2-4}$ as demonstrated in the Iowa gambling task (IGT), a test that simulates real-life decision making. ${ }^{5}$ In this test, the goal is to win as much as possible by discerning which of the two decks of cards are advantageous and which two are disadvantageous. Previous research on the IGT has shown that patients with chronic pain score lower and/or switch more frequently between decks compared to healthy controls (HCs). ${ }^{2-4}$

The IGT was introduced as a test of the somatic marker hypothesis. ${ }^{5}$ This hypothesis states that when facing ambiguous decisions, cognitive processes are insufficient in guiding choices. ${ }^{6,7}$ Instead, autonomic physiological reactions, such as skin conductance responses (SCRs) that are learned to be associated with a specific outcome, are engendered in the body and are relayed to the brain where they give rise to an
Department of Neuroscience, Medical Faculty, Pb 8905, Norwegian University of Science and Technology (NTNU), 749I Trondheim, Norway Tel +47735 51352

Email asta.haberg@ntnu.no 
emotion-guided decision. ${ }^{7}$ This is called a somatic marker. In healthy subjects, increased anticipatory SCRs are present before choosing cards from the disadvantageous decks of cards in the IGT (see Figures 1 and 2 for an illustration of the model). ${ }^{8}$ This is not found in subjects with lesions in the ventromedial prefrontal cortex, who have impaired decisionmaking skills, but who are otherwise spared intellect. ${ }^{8,9}$ Similarly, the reduced decision-making ability in patients with chronic pain may arise from a lack of anticipatory SCR generation, but this has not yet been investigated.

As far as we know, there is also a lack of knowledge as to whether impaired performance on the IGT correlates with changes in the cerebral morphology of patients with chronic pain. This may be expected, since several brain regions involved in decision making are reported to have altered morphology in chronic pain patients. ${ }^{10}$ Decision making relies on many brain regions for generating, relaying, and interpreting SCR and/or other somatic signals. The winner of any competing signals influences the final choice. ${ }^{7}$ This process is dependent on trigger structures (the amygdala and ventromedial prefrontal cortex), effector structures (such as the hypothalamus, periaqueductal gray area, nucleus accumbens, and neurotransmitter brainstem nuclei), sensory structures (sensory brain stem nuclei), and processing structures (such as the insula and somatosensory cortices), as well as memory structures (for instance, the hippocampus and dorsolateral prefrontal cortex). ${ }^{7}$ In patients with chronic pain, gray matter volume reductions have been reported in all these mentioned structure groups, most consistently in the trigger (ventromedial prefrontal cortex), processing (insula), and memory (dorsolateral prefrontal cortex) structures. ${ }^{10-12}$

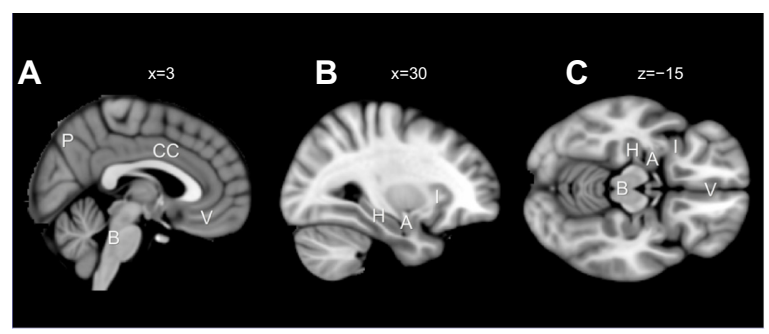

Figure I Decision-making structures important during the lowa gambling task. Notes: Trigger structures are the $(\mathbf{A}$ and $\mathbf{C}) \mathrm{V}$, and (B and $\mathbf{C}) \mathrm{A}$. The A mainly works as a trigger structure for the impulsive system, while the $V$ is a trigger structure for the reflective system. Important supporting structures for the $V$ are the dorsolateral prefrontal cortex (not shown) and (B and $\mathbf{C}$ ) $\mathrm{H}$. Effector structures include the hypothalamus, nucleus accumbens, and periaqueductal gray area, while sensory structures include the sensory brain stem nuclei such as the parabrachial nucleus and neurotransmitter nuclei. For effector structures and sensory structures, the figure only displays the (A and $\mathbf{C}$ ) B. Important structures for processing information from the sensory structures are the (B and $\mathbf{C}) \mathrm{I}$, the $(\mathbf{A}) \mathrm{CC},(\mathbf{A})$ the $\mathrm{P}$, as well as the somatosensory cortices (not shown). $X$ and $Z$ give the location of the two saggital and one transverse planes in the illustration.

Abbreviations: $\mathrm{P}$, precuneus; $\mathrm{B}$, brainstem; $\mathrm{CC}$, cingulated cortex; $\mathrm{V}$, ventromedial prefrontal cortex; $\mathrm{H}$, hippocampus; $\mathrm{A}$, amygdala; I, insula.
The overall intention of this study was to explore and compare differences and associations between IGT behavior, autonomic signals, and brain structure volumes in patients with chronic pain and matched HCs. The main aim was to examine whether patients' impaired decision making is associated with a lack of anticipatory autonomic physiological reactions. We predicted, based on the previously reported deficits in performance on the IGT in chronic pain patients, ${ }^{2-4}$ and on the somatic marker hypothesis, that the patient group would lack the anticipatory SCR before picking cards from the disadvantageous decks. To elucidate possible contributions of other autonomic physiological reactions on IGT performance in the patient group, heart rate (HR) and blood pressure were also measured. The secondary aim was to explore the associations between IGT performance and brain structure volumes. We anticipated that reduced brain structure volumes would be related to impaired IGT performance in patients with chronic pain.

\section{Methods}

The study was approved by the Regional Committee for Medical Research Ethics and the Norwegian Social Sciences Data Service, and was performed in accordance with their requirements and the Declaration of Helsinki. Written informed consent was obtained from all participants.

\section{Materials}

Twenty subjects ( 16 females) with chronic pain were recruited during consultations in a university pain clinic, and 20 (18 females) age- and education-matched HCs were recruited from the local community. Prior to inclusion, the patients had to report a 6-month average pain intensity of $\geq 4$ on the Verbal Rating Scale, with scores ranging from $0-10 .{ }^{13}$ The included patients also had to be in a chronic pain state, which corresponds to Verbal Rating Scale scores $\geq 4$ for at least 6 months. ${ }^{14}$

All participants were offered a monetary compensation of 400 Norwegian Kroner (NOK) (approximately USD $\$ 65)$ and pictures from their morphological brain scan. Psychiatric and neurological disorders, known traumatic brain injuries (13 Glasgow coma scale score at the time of injury), or magnetic resonance imaging (MRI) contraindications were used as exclusion criteria. A diagnosis of mild or moderate depression did not warrant exclusion in any of the groups. Furthermore, patients with a high consumption of analgesics were excluded ( $>180 \mathrm{mg}$ of codeine or its equivalent per 24 hours, 24-hour continuous benzodiazepine treatment, or use of carisoprodol). All participants 

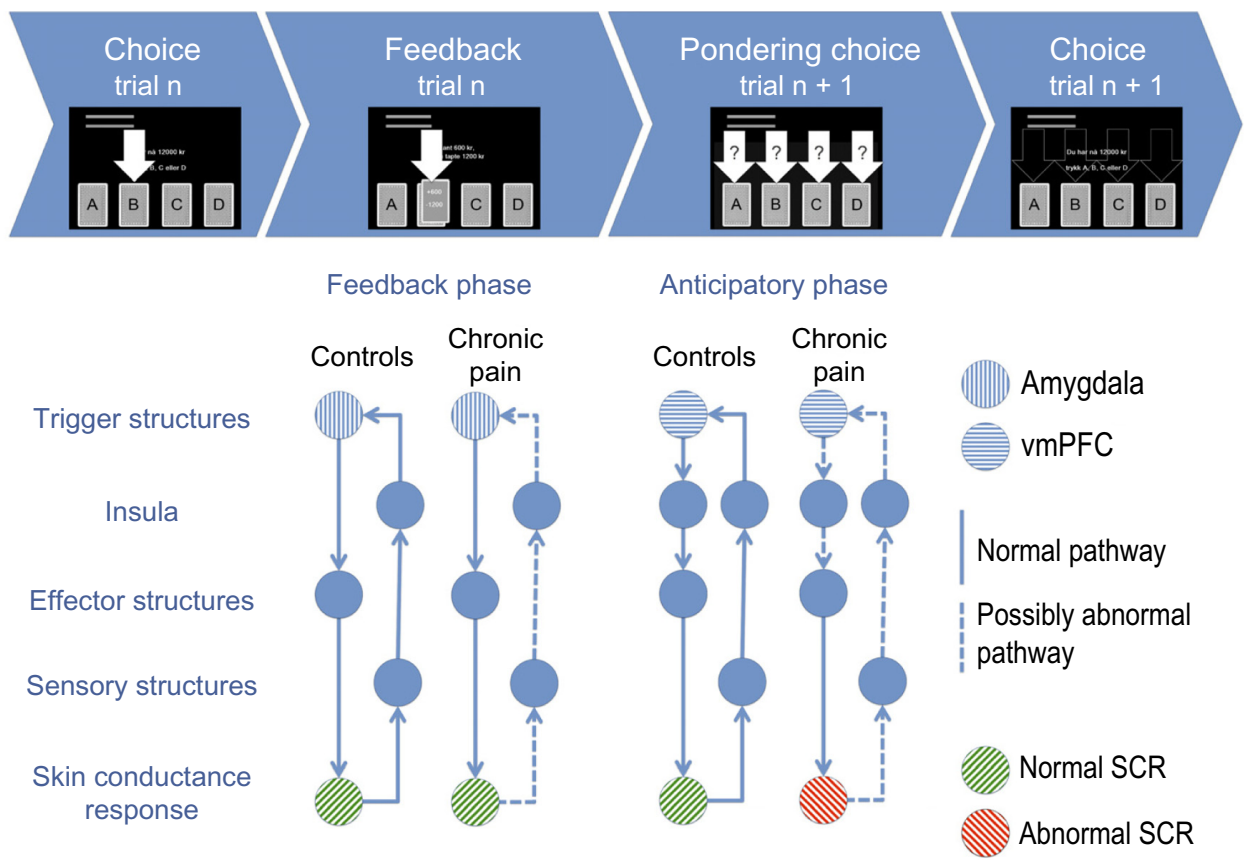

Figure 2 The generation of SCR during the IGT in the PCP and HC groups in the present study in light of the somatic marker hypothesis.

Notes: The schematic representation of the neuronal structures involved in generating and utilizing SCR during the IGT is from the somatic marker hypothesis. ${ }^{7}$ The top row shows the phases of the IGT. After choice $\mathrm{n}$, the subject is presented with a reward and possibly a punishment (feedback phase). This visual feedback generates a feedback SCR. The subject ponders choice $\mathrm{n}+\mathrm{I}$, which generates an anticipatory SCR (anticipatory phase). Anticipatory SCRs are interpreted by the brain and influence choice $\mathrm{n}+\mathrm{I}$. The flow charts show the structures involved in generating SCRs and interpreting them during the IGT in HCs and in PCP. The arrows indicate the direction of information flow in a continuous process, initiated by the two trigger structures. The amygdala is more important as a trigger structure in the feedback phase due to its role in the impulsive system. The ventromedial prefrontal cortex is more important as a trigger structure in the anticipatory phase, and it triggers effector structures via the insula. The dotted lines indicate pathways that the current study suggests are abnormal in PCP, since they managed to generate normal feedback SCRs, but not normal anticipatory SCRs during pondering.

Abbreviations: SCR, skin conductance response; IGT, lowa gambling task; PCP, patients with chronic pain; HC, healthy control; n, choice number; vmPFC, ventromedial prefrontal cortex.

reported being right-handed, and they were assessed with the Edinburgh Handedness Inventory ${ }^{15}$ (patients: $0.82 \pm 0.21$ [mean \pm standard deviation]; controls: 0.91 \pm 0.16 ).

One patient was excluded due to a neurological disease that was discovered after inclusion, and one patient and one control were excluded due to technical problems during the IGT presentation. Thus, the groups included in the final analysis consisted of 18 patients with chronic pain (15 females) and 19 HCs (17 females).

Of the 18 included chronic pain patients, eleven were classified as having pain of musculoskeletal etiology, five of idiopathic etiology, two with visceral etiology, and none with neuropathic pain.

\section{Data collection}

\section{Procedure}

On day 1, the participants underwent MRI scanning. On day 2 , the participants filled out questionnaires measuring pain and completed neuropsychological tests to assess their general intelligence, depression level, working memory and effort, and finally performance on the IGT during neurophysiological monitoring.
The IGT testing began with the subjects visiting the lavatory and washing their hands to ensure good SCR readings. Following that, various autonomic measuring equipment were attached (see below). Instructions for the computerized version of the IGT ${ }^{16}$ were read to the subjects by the researcher (NAE) while the game was demonstrated. The subjects were then left alone in the room and monitored by closedcircuit camera and microphone by the researcher. They were instructed to relax while an on-screen clock counted down from five minutes, and they were then instructed to commence with the task. They began to choose cards by pressing keyboard keys labeled A, B, C, and D, which corresponded to the labeling of the card decks on the screen.

Room temperature was consistently maintained between $22^{\circ} \mathrm{C}-26^{\circ} \mathrm{C}$, and this was confirmed before testing with an electronic thermometer (Digitron 2088T, Elektron Technology, Cambridge, UK).

\section{lowa gambling task (IGT)}

We used a modified computerized version of the original IGT, which is similar to the version described by Bechara et al. ${ }^{16}$ To avoid confusion about the value of foreign currencies, 
all USD values from the original IGT were converted to local currency (NOK). Subjects chose cards from four decks (A, $\mathrm{B}, \mathrm{C}$, and D) with the goal of winning as much as possible. In each deck, there are varying amounts of rewards and punishments, with decks A and B offering a fixed gain of \$100, and decks $C$ and D offering a fixed gain of $\$ 50$. The losses vary in frequency, with a $10 \%$ loss in decks B and D, and a $50 \%$ loss in decks $\mathrm{A}$ and $\mathrm{C}$. This results in an average gain or loss after ten cards, with a $\$ 250$ loss for cards from decks $A$ and $B$, and a \$250 gain for decks C and D. Decks C and D were thus the advantageous decks, and $\mathrm{A}$ and $\mathrm{B}$ were the disadvantageous decks. On the computer display of the four card decks, a red bar indicating the amount of debt and a green bar indicating the amount of winnings were presented.On the same screen, updated instructions were presented to the subjects in Norwegian ("You now have X NOK", "Press A, B, C, or D", "You won Y NOK", and on some trials "but you lost Z NOK", where $\mathrm{X}, \mathrm{Y}$ and $\mathrm{Z}$ were currency amounts) (Figure 3). The IGT has been described in more detail elsewhere. ${ }^{16}$

We randomized the on-screen position and naming of the different decks to avoid bias from naming or placement on the screen (see Figure 3). ${ }^{17}$ Furthermore, we shuffled the decks of cards between subjects. Randomization of the card order increases the robustness of averaging the autonomic

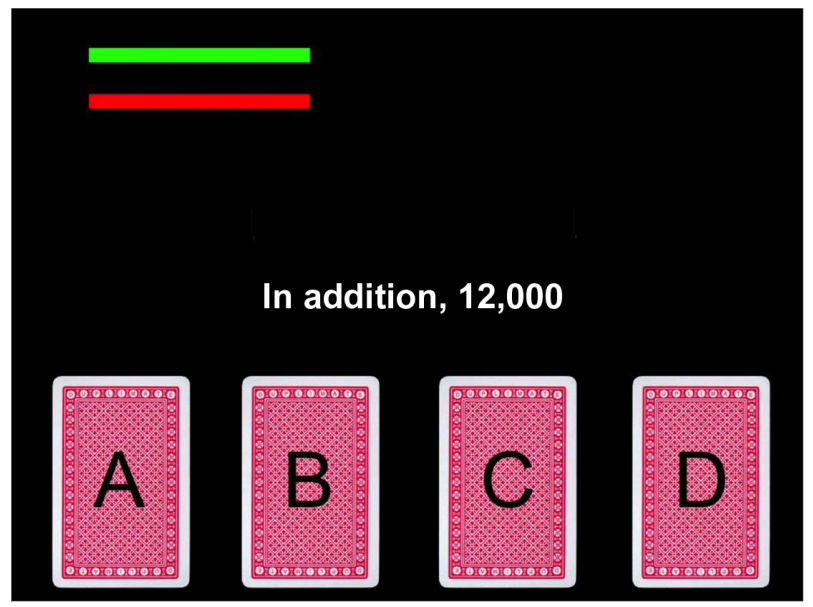

Figure 3 The lowa gambling task.

Notes: The lowa gambling task is designed to test decision making. The figure illustrates the starting screen on a computerized version developed by the first author. The four decks from which subjects can choose have different and fixed rewards, while punishment frequencies vary between the decks, and punishment size also varies within the decks. From these characteristics, two decks are disadvantageous over time, while two decks are advantageous over time. Decision-making ability is scored by the number of cards the subject draws from the advantageous decks minus the cards drawn from the disadvantageous decks. The white text provides instructions to the subject in Norwegian ("You now have $\$ 2,000 /$ press A, B, C, or D"). The green bar displays the amount of money the subject has, while the red bar displays the total sum of money the subject has borrowed to play. In this version, the placement of the four decks was randomized on the screen for each subject, and renamed A-D from left to right. In this article A-D refers to the decks by the classic nomenclature of the lowa gambling task, not the letters displayed to the subjects. signal, and it also rules out the effect of a specific card order on somatic marker generation. Randomization was obtained by block randomization in blocks of ten cards, keeping the original punishment and reward frequencies as described by Bechara et al. ${ }^{5}$

The interval from one choice to the next was set to a minimum of 6.5 seconds, and it ended when the subject chose a card. The IGT has been criticized for allowing certain decks to run out of cards, ${ }^{18}$ thus reducing its sensitivity to detecting impairment in decision making. Because of this, we set all decks to contain 100 cards, which is equal to the total amount of cards that the subject was allowed to draw.

\section{Autonomic measures}

As a measure of the state and balance of the autonomic nervous system, several autonomic measurements were made. Skin sweat gland activity (ie, SCR), HR (ie, R wave to R wave intervals $[R R]$ ) from electrocardiogram (ECG) (PowerLab unit, ADInstruments, Dunedin, New Zealand) recordings, and blood pressure from the finger manometer were recorded during IGT performance. The ECG data was used to calculate both $H R$ and HR variability. HR variability was calculated based on the HR, according to current recommendations. ${ }^{19} \mathrm{SCR}, \mathrm{RR}$ intervals, and blood pressure were used in event-related analyses (autonomic activity directly preceding or following choices). The average HR, HR variability, and blood pressure were calculated separately for the 5-minute baseline period before the IGT ("Baseline") and for the entire IGT period ("Activity"). In addition the individual's change $(\Delta)$ in these measurements from Baseline to Activity was calculated, for example:

$$
\text { Baseline HR }- \text { Activity HR }=\Delta \mathrm{HR} \text {. }
$$

\section{Sample characteristics}

Pain

Pain intensity was assessed using the validated Norwegian translation of the Brief Pain Inventory (BPI). ${ }^{20,21}$ The BPI assesses the intensity of pain during the last 24 hours using a 0-10 numerical rating scale.

\section{Depression level}

Since major depressive disorder is known to affect decision making, ${ }^{22}$ depression levels were scored with the Beck Depression Inventory-II to enable the correction of IGT scores. ${ }^{23}$

\section{Working memory}

Working memory function is necessary for normal IGT performance, ${ }^{24}$ and reduced working memory performance 
has been reported in chronic pain samples. ${ }^{25}$ Thus, all subjects completed the Letter-Number Sequencing subtest of the Wechsler Adult Intelligence Scale III, a standard two-back test, and a visual version of the Paced Auditory Serial Addition Test. ${ }^{26}$ These three tasks cover various aspects of working memory, ranging from simple storage of information in working memory, to manipulation of the stored information.

\section{Hardware}

For the autonomic measurements, all data were collected using Chart (version 5.5; ADInstruments, Dunedin, New Zealand) and sampled at $1 \mathrm{kHz}$. The computer was fed data from PowerLab 16/30 (ADInstruments), which got user input data and card information data via a serial output/ parallel input cable (Leteng AS, Oslo, Norway). The SCR was measured with finger electrodes with a dedicated preamplifier (MLT116F and ML135; ADInstruments). Respiration (Thermistor; Sleepmate ${ }^{\circledR}$ Technologies, Glen Burnie, MD, USA) and one-lead ECG (lead II) were also measured. Continuous finger blood pressure was measured (Finometer ${ }^{R}$ PRO, internal sampling at $200 \mathrm{~Hz}$; Finapres Medical Systems BV, Amsterdam, the Netherlands). Recordings of blood pressure and SCR were done on the left (nonmoving) hand to reduce motion artifacts.

\section{Image acquisition}

Scanning was performed on a 3T Siemens Trio scanner with a 12-channel Head Matrix Coil (Siemens AG, Munich, Bavaria, Germany). Foam pads were used to minimize head motion. One T1-weighted three-dimensional volume measurement was acquired (repetition time $[\mathrm{TR}]=2,300 \mathrm{~ms}$; echo time $[\mathrm{TE}]=2.88 \mathrm{~ms}$; inversion time $[\mathrm{TI}]=900 \mathrm{~ms}$; flip angle $=9^{\circ}$; field of view $[\mathrm{FOV}]=526$; slices $=160$; slice thickness $=1.2$ $\mathrm{mm}$; in-plane resolution of $1.0 \mathrm{~mm} \times 1.0 \mathrm{~mm}$ ). No morphological abnormalities were revealed in any of the participants.

\section{Analysis}

\section{IGT measurements}

The IGT score was calculated as the number of advantageous (cards chosen from decks $\mathrm{C}$ and D) minus disadvantageous choices (cards chosen from decks A and B).

Patients with chronic pain have been shown to have reduced persistence during IGT performance (ie, they switch more often between the four decks of cards) than controls. ${ }^{2,4}$ IGT switching was calculated as the frequency at which a subject switched from drawing from an advantageous or disadvantageous deck choice, to drawing from the other type of deck choice. For example, "A, A" and "A, B" were not counted as a switch, but "A, C" and "A, D" were counted as a switch.

For IGT score and switching, total score and total switching was calculated for the whole test (cards 1 to 100). Additionally, score and switching was calculated for the learning phase of the test (cards 1 to 40) and the performance phase of the test (cards 41 to 100), as the processes underlying decision making have been shown to differ in the first 40 versus the last 60 cards in controls ${ }^{27}$ and in patients with chronic pain. ${ }^{2}$

\section{Event-related autonomic analysis}

Previous research on anticipatory SCR during the IGT has used a variety of methods for calculating the SCR. ${ }^{28-31} \mathrm{We}$ measured both anticipatory responses (5-0 seconds prior to making a choice) and feedback responses ( $0-5$ seconds after making a choice) for SCR. SCRs were calculated in a manner similar to that reported by Bechara et $\mathrm{al}^{32}$ (ie, the integral of the detrended skin conductance level curve, or the area under the curve, was obtained, with the skin conductance level recorded at the beginning of the integral serving as the baseline).

We used the same method to calculate the integral of systolic blood pressure and heart RR intervals in the 5 seconds prior to making a choice and after making a choice.

For the analysis of blood pressure levels, the delay from true aortic blood pressure to the pulse signals measured in the hand was assumed at a fixed $250 \mathrm{~ms}$ delay. Automatic calibration of the blood pressure monitoring equipment and ectopic heart beats were identified by manual inspection of the data, and any RR or blood pressure intervals that included such events were excluded from the analysis.

Postchoice SCRs were analyzed to assess whether SCR generation following punishment or reward was similar in the control and patient groups. For this analysis, SCRs after making a choice were grouped based on whether the card actually punished the subject or not ( $50 \%$ of cards in decks $\mathrm{A}$ and $\mathrm{C}$ and $10 \%$ of cards in decks B and D punished the subject). The integral was analyzed in the 5-second period after making a choice; otherwise, they were similar to anticipatory SCR calculations.

Analyses at the event level (ie, integrals for a period prior to making a card selection for a given subject) and HR variability analyses were done in Chart 7.0 (ADInstruments).

\section{Cardiac autonomic regulation}

Analysis of normalized low frequency (LF) power (0.04-0.15 $\mathrm{Hz} \mathrm{ms}^{2}$ ), normalized high frequency (HF) power $\left(0.15-0.4 \mathrm{~Hz} \mathrm{~ms}^{2}\right)$, and the LF to $\mathrm{HF}$ ratio ( $\left.\mathrm{LF} / \mathrm{HF}\right)$ were performed in the frequency domain (LabChart 7.0; ADInstruments) 
(Welch window, 1,024 data points, and segment overlap, 50\%). The maximal frequency was set to $0.5 \mathrm{~Hz}$.

Artifacts were excluded from the analyses, and RR intervals were estimated from the intervals noted before and after the ectopic heartbeats occurred. The recordings were inspected manually and corrected when necessary.

HR, HR variability, and blood pressure were calculated as the average for the 5-minute resting period before the start of the IGT (for example, "Baseline HR"), for the duration of the IGT (for example, "Activity HR"), and for each group's mean absolute change from Baseline to Activity across all cardiac autonomic measures (for example, " $\Delta \mathrm{HR}$ ").

\section{MRI analysis}

The volumetric assessment of subjects' T1-weighted brain MRI volumes was performed using NeuroQuant software (CorTechs Labs, Inc., La Jolla, CA, USA). This software enables automated analysis of T1-weighted brain volumes and provides a morphology report on the volume of the total cortical gray matter, as well as of the ventricles, brainstem, cerebellum, and some subcortical structures (hippocampus, amygdala, caudate, putamen, pallidum, thalamus, nucleus accumbens, and brainstem), which was corrected for intracranial volume. ${ }^{33}$ For each structure, the volumes of the structures in the right and left hemisphere were combined. The volumes of the structures of interest (total cortical gray matter, amygdala, hippocampus, brainstem, and nucleus accumbens) were compared between groups and correlated with total IGT scores, pain, and autonomic data.

\section{Statistical analysis}

PASW Statistics 18.0 (IBM Corporation, Armonk, NY, USA) was used for all statistical analyses.

Two-tailed, unpaired Student's $t$-tests were used to identify the differences between groups on demographic, depression level, pain, and neuropsychological measures, as well as on IGT scores, brain structure volumes, and autonomic activity. Cohen's $d$ was calculated and classified as small $(d=0.15-$ $0.40)$, medium $(d=0.40-0.75)$, and large $(d>0.75)$.

Paired Student's $t$-tests were used for all within-groups analyses of event-related or cardiac autonomic measures. Spearman's rank-order correlation was performed to assess the relationships between the IGT total score, the IGT total switching, pain level (evaluated using the BPI) before the IGT, and event-related autonomic data, as well as between the IGT total score and brain structure volumes. Possible confounding effects of depression levels on the IGT score were investigated with Spearman's partial correlation. The significance threshold was set to $P \leq 0.05$ (two-tailed) for both Student's $t$-tests and Spearman's correlations.

A mixed-design analysis of variance (ANOVA) ("splitplot ANOVA") was used to investigate the interactions between the three autonomic measures related to choosing from the advantageous versus disadvantageous decks and subject group (chronic pain patients versus HCs). Outliers were identified as studentized residuals $\geq \pm 3$ standard deviations. Simple main effects were investigated where significant interactions were found. Effect sizes were calculated as partial eta squared $\left(\eta p^{2}\right)$, and they were classified as small $(>0.01)$, medium $(>0.06)$, or large $(>0.14)$.

All data are given as the mean \pm standard deviation.

\section{Results}

\section{Participants}

As shown in Table 1, there were no differences in age, length of education, or the sex distribution between the patients and control groups. The patient group scored significantly higher on both the average pain for the last 24 hours and average pain at the time of testing than did the controls. (Table 1).

There were no significant differences on the working memory tests (Table 1).

\section{lowa gambling task behavior}

As shown in Table 2, the IGT total score was not different between the patients and controls. There were also no differences in the IGT score for the learning or performance phases of the IGT between the groups (Table 2).

During the entire IGT period, there was a trend toward increased switching in the patient group compared to the

Table I Demographic, clinical, and working memory measures in patients with chronic pain and matched healthy controls

\begin{tabular}{lllll}
\hline & PCP & HCs & P & d \\
\hline Education & $4.67 \pm 2.4$ & $5.26 \pm 2.7$ & 0.484 & 0.23 \\
Sex & $18(3 \mathrm{male})$ & $19(2 \mathrm{male})$ & 0.597 & 0.18 \\
Age & $38.5 \pm 7.1$ & $38.4 \pm 7.0$ & 0.955 & 0.02 \\
$\begin{array}{l}\text { Pain NRS (prior 24 hour } \\
\text { average) }\end{array}$ & $6 \pm 1.64$ & $1 \pm 1.29$ & $0.000^{*}$ & 3.39 \\
Pain NRS (at testing) & $4.11 \pm 1.49$ & $0.05 \pm 0.23$ & $0.000^{*}$ & 3.86 \\
$\begin{array}{l}\text { Depression level } \\
\text { Working memory measures }\end{array}$ & $14.2 \pm 8.6$ & $1.9 \pm 2.4$ & $0.000^{*}$ & 1.96 \\
$\quad$ & & & & \\
$\quad \begin{array}{l}\text { 2-btter-number sequencing score } \\
\quad \text { PVSAT score }\end{array}$ & $9.2 \pm 2.2$ & $9.9 \pm 2.0$ & 0.313 & 0.34 \\
\hline
\end{tabular}

Notes: Depression level was determined with the Beck Depression Inventory II. Numbers are the numbers are the mean values \pm standard deviation within groups of PCP with a pain self-rating of $\geq 4 / 10$ for $\geq 6$ months and in their healthy controls. Statistical differences between groups were explored with a two-tailed, two-sample Student's $t$-test. Significance on the Student's $t$-test is marked with $*$ for $P \leq 0.05$. Effect size is calculated as Cohen's $d$.

Abbreviations: PCP, patients with chronic pain; HCs, healthy controls; NRS, Numerical Rating Scale; PVSAT; Paced Visual Serial Addition Test. 
Table 2 IGT total scores and switching in the learning phase ( $\mathrm{I}-40$ cards) and performance phase (4I-I00 cards), and brain volumes in $\mathrm{PCP}$ and matched $\mathrm{HCs}$

\begin{tabular}{lrrll}
\hline & PCP & HCs & \multicolumn{1}{l}{$\boldsymbol{P}$} & \multicolumn{1}{l}{$\boldsymbol{d}$} \\
\hline IGT score & $5.2 \pm 28.6$ & $5.7 \pm 31.0$ & 0.959 & 0.02 \\
IGT score, learning phase & $-7.4 \pm 10.7$ & $-9.2 \pm 13.3$ & 0.665 & 0.14 \\
IGT score, performance phase & $-0.3 \pm 26.9$ & $1.6 \pm 27.4$ & 0.830 & 0.07 \\
Switch total & $0.35 \pm 0.15$ & $0.26 \pm 0.15$ & 0.080 & 0.59 \\
Switch, learning phase & $-6.13 \pm 0.16$ & $-6.17 \pm 0.17$ & 0.472 & 0.24 \\
Switch, performance phase & $-6.17 \pm 0.18$ & $-6.29 \pm 0.16$ & $0.03 I^{*}$ & 0.74 \\
Brain volume, \% of ICV & & & & \\
$\quad$ Total cortical gray matter & $32.75 \pm 2.81$ & $33.93 \pm 2.47$ & 0.209 & 0.44 \\
Hippocampus & $0.53 \pm 0.04$ & $0.54 \pm 0.05$ & 0.872 & 0.06 \\
Amygdala & $0.25 \pm 0.02$ & $0.25 \pm 0.02$ & 0.556 & 0.20 \\
Nucleus accumbens & $0.07 \pm 0.01$ & $0.08 \pm 0.01$ & $0.034 *$ & 0.75 \\
Brainstem & $1.62 \pm 0.15$ & $1.57 \pm 0.11$ & 0.293 & 0.36 \\
\hline
\end{tabular}

Notes: Numbers are the mean values \pm standard deviation within groups of PCP with a pain self-rating of $\geq 4 / 10$ for $\geq 6$ months and in their healthy controls Cerebral volume is the combined volume of the two hemispheres in the \% of ICV. Statistical differences between groups were explored with a two-tailed, two-sample Student's $t$-test. Significance of the Student's $t$-test is marked with * for $P \leq 0.05$. Effect size is calculated as Cohen's $d$.

Abbreviations: IGT, lowa gambling task; PCP, patients with chronic pain; HCs, healthy controls; ICV, intracranial volume.

control group. In the performance phase of the IGT, the chronic pain patients switched significantly more often than did the HCs (Table 2).

There were no significant correlations between the different IGT scores and pain level before the test in any of the groups (Table 3). These correlations remained nonsignificant after adjusting for depression scores (results not shown).

\section{Autonomic activation during decision making SCR}

A mixed-design ANOVA (group [chronic pain patient or control] $\times$ deck [advantageous or disadvantageous]) on the
SCR before choices showed a weak, nonsignificant main effect for group, no main effect for deck, but a significant group $\times$ deck interaction with a large effect size (Table 4 and Figure 4). There was one outlier in the patient group, but the group $\times$ deck interaction remained when excluding the outlier $\left(F[1,33]=5.227, P=0.029, \eta_{p}^{2}=0.137\right)$.

The SCRs before choosing from advantageous and disadvantageous decks were significantly different, with a large effect size observed within the control group (Table 4 and Figure 5). In the patient group, there was no difference in SCRs before choosing from advantageous and disadvantageous decks (Table 5 and Figure 4). For disadvantageous decks, the SCR was significantly higher in controls than in patients with a large effect size $(F[1$, $\left.34]=6.581, P=0.015, \eta_{p}^{2}=0.162\right)$. There was no such group difference in SCR before the advantageous deck choices (Figure 2).

For HCs, there was a significant correlation between the SCR before the disadvantageous deck choices and the IGT total score (Spearman's rho $=0.568, P=0.011$ ). This was not found in the patient group. Rather, the SCRs before the disadvantageous and advantageous deck choices were made were correlated with each other in the chronic pain group (Spearman's rho $=0.539, P=0.026$ ). Except for the aforementioned results, no correlations were found in the chronic pain group between SCR and IGT behavior, or between SCR and pain level (Table 3). None of these correlations changed in significance after adjusting for depression level (results not shown).

There were no differences in the postchoice SCRs between receiving a punishment and a no-punishment card within the patient or control groups, or between the groups (Table 5).

Table 3 Correlations between pain level, the different IGT scores, and SCR before and during the IGT in PCP and matched HCs

\begin{tabular}{|c|c|c|c|c|c|c|c|c|c|c|}
\hline & PCP & & & & & HCs & & & & \\
\hline & $\begin{array}{l}\text { Pain } \\
\text { level }\end{array}$ & $\begin{array}{l}\text { IGT } \\
\text { score }\end{array}$ & $\begin{array}{l}\text { IGT } \\
\text { switching }\end{array}$ & $\begin{array}{l}\text { SCR } \\
\text { Before } \\
\text { advantageous } \\
\text { choices }\end{array}$ & $\begin{array}{l}\text { SCR } \\
\text { Before } \\
\text { disadvantageous } \\
\text { choices }\end{array}$ & $\begin{array}{l}\text { Pain } \\
\text { level }\end{array}$ & $\begin{array}{l}\text { IGT } \\
\text { score }\end{array}$ & $\begin{array}{l}\text { IGT } \\
\text { switching }\end{array}$ & $\begin{array}{l}\text { SCR } \\
\text { Before } \\
\text { advantageous } \\
\text { choices }\end{array}$ & $\begin{array}{l}\text { SCR } \\
\text { Before } \\
\text { disadvantageous } \\
\text { choices }\end{array}$ \\
\hline Pain level & I* & & & & & I* & & & & \\
\hline IGT score & -0.388 & I* & & & & -0.151 & I* & & & \\
\hline IGT switching & 0.262 & -0.009 & I* & & & 0.172 & -0.277 & I* & & \\
\hline $\begin{array}{l}\text { SCR before } \\
\text { advantageous } \\
\text { choices }\end{array}$ & -0.02 & 0.103 & 0.091 & $\mathrm{I}^{*}$ & & 0.172 & 0.184 & -0.119 & I* & \\
\hline $\begin{array}{l}\text { SCR before } \\
\text { disadvantageous } \\
\text { choices }\end{array}$ & 0.044 & 0.063 & 0.203 & $0.539 *$ & I* & 0.215 & $0.568^{*}$ & 0.151 & 0.253 & $I^{*}$ \\
\hline
\end{tabular}

Notes: Numbers are Spearman's rho in PCP with a pain self-rating of $\geq 4 / 10$ for $\geq 6$ months and in their healthy controls. Statistical differences within groups were explored with a two-tailed Spearman's rank-order correlation. Significance of the Student's t-test is marked with * for $P \leq 0.05$.

Abbreviations: IGT, lowa gambling task; SCR, skin conductance response; PCP, patients with chronic pain; HCs, healthy controls. 
Table 4 Results of the mixed ANOVA (group $\times$ choice type) analyses of the autonomic SCR, heart rate (RR), and BP before choosing from the disadvantageous or the advantageous decks in $\mathrm{PCP}$ and matched $\mathrm{HCs}$

\begin{tabular}{|c|c|c|c|}
\hline & $\boldsymbol{F}$ & $\mathbf{P}$ & $\eta_{p}^{2}$ \\
\hline \multicolumn{4}{|l|}{ SCR } \\
\hline Group & $F(I, 34)=2.869$ & 0.099 & 0.078 \\
\hline Deck & $F(I, 34)=1.005$ & 0.323 & 0.029 \\
\hline Group $\times$ deck & $F(1,34)=6.195$ & $0.018^{*}$ & 0.154 \\
\hline \multicolumn{4}{|l|}{ RR } \\
\hline Group & $F(1,32)=1.917$ & 0.176 & 0.057 \\
\hline Deck & $F(I, 32)=2.755$ & 0.107 & 0.079 \\
\hline Group $\times$ deck & $F(I, 32)=0.542$ & 0.467 & 0.017 \\
\hline \multicolumn{4}{|l|}{ BP } \\
\hline Group & $F(I, 32)=1.753$ & 0.802 & 0.002 \\
\hline Deck & $F(1,32)=1.916$ & 0.176 & 0.056 \\
\hline Group $\times$ deck & $F(I, 32)=I .382$ & 0.249 & 0.041 \\
\hline \multicolumn{4}{|l|}{ Simple main effects } \\
\hline \multicolumn{4}{|l|}{$S C R$} \\
\hline HC group & $F(I, \mid 8)=5.349$ & $0.033^{*}$ & 0.229 \\
\hline PCP group & $F(I, I 6)=1.363$ & 0.260 & 0.079 \\
\hline Disadvantageous decks & $F(I, 34)=6.58 I$ & $0.015^{*}$ & 0.162 \\
\hline Advantageous decks & $F(I, 34)=0.374$ & 0.545 & 0.011 \\
\hline
\end{tabular}

Notes: Relationship between group status and anticipatory autonomic activation among PCP with a pain self-rating of $\geq 4 / 10$ for $\geq 6$ months and in their healthy controls. The mixed ANOVA is by group (PCP or $\mathrm{HCs}$ ) $\times$ deck type (advantageous or disadvantageous) for each of the three autonomic measures used in the study (SCR, RR, and BP). One patient was excluded from all SCR analyses due to technical problems with the recordings. Two controls were excluded from all BP and heart rate analyses because of excessive amount of extrasystoles. Measures are integrals of autonomic measures in the 5 seconds preceding the subject's choice of card, which were either classified as advantageous or disadvantageous. Significance of the Student's $t$-test is marked with * for $P \geq 0.05$.

Abbreviations: ANOVA, analysis of variance; SCR, skin conductance response; $R R$, $R$ wave to $R$ wave interval; $B P$, systolic blood pressure; $P C P$, patients with chronic pain; HCs, healthy controls.

\section{RR intervals}

A mixed-design ANOVA on the RR integral before choices (group [chronic pain patient or control] $\times$ deck [advantageous or disadvantageous]) showed no main effect for group, deck, or the group $\times$ deck interaction (Table 4$)$. There were no outliers in any group.

\section{Blood pressure}

The mixed-design ANOVA on the blood pressure integral before choices (group [chronic pain patient or control] $\times$ deck [advantageous or disadvantageous]) showed no main effect for group, deck, or the group $\times$ deck interaction (Table 4). There was one outlier in the control group, but removing this did not alter the results.

\section{Cardiac autonomic regulation}

The Student's $t$-tests showed no significant group differences for the Baseline HR or Baseline HR variability measures (LF/ HF, LF, or HF), or the Baseline blood pressure between the patient and the control groups, and all of the effect sizes were small. There was also no significant group difference for the change from Baseline to Activity on any of the cardiac autonomic measures. However, there were medium effect sizes for the group differences on $\Delta \mathrm{HR}, \Delta \mathrm{LF} / \mathrm{HF}$, and $\Delta \mathrm{HF}$ (Table 6).

\section{Brain structure volumes}

As shown in Table 2, the nucleus accumbens volume was significantly reduced in the chronic pain group. For the other
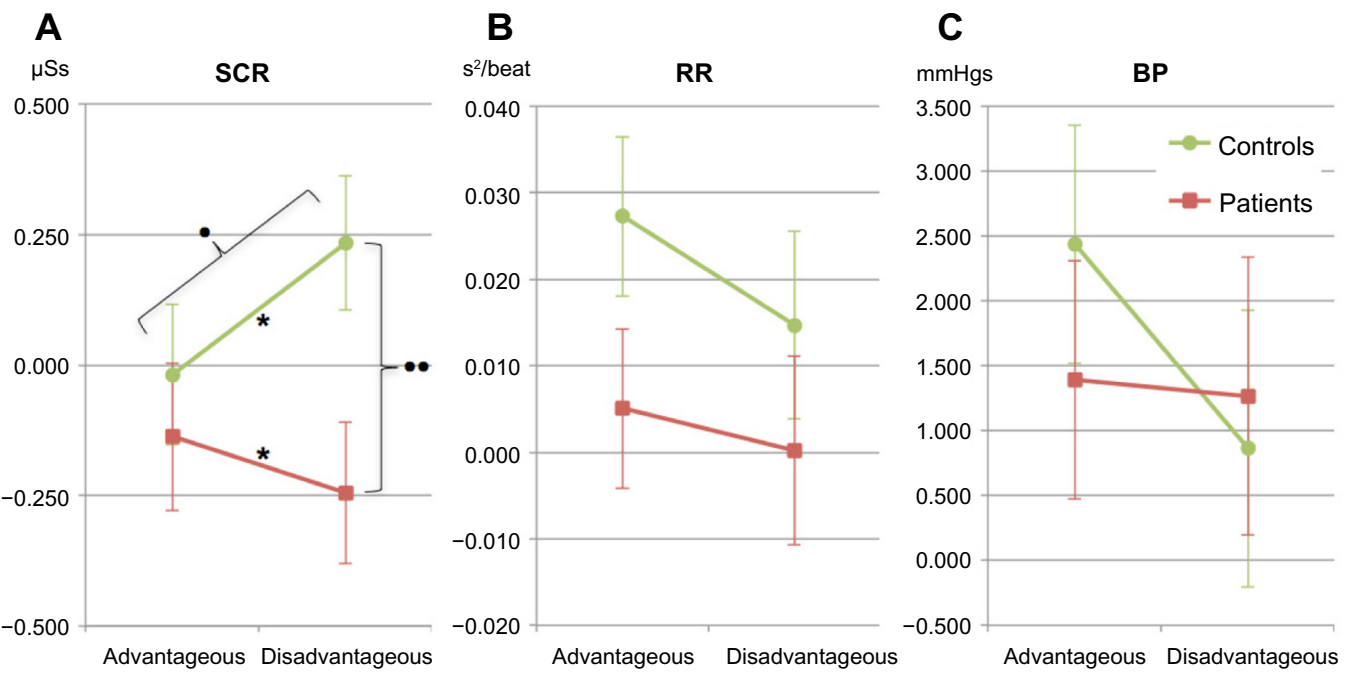

Figure 4 Autonomic measures from the anticipatory phase of the lowa gambling task before drawing from either the advantageous or disadvantageous decks. Notes: The autonomic measures were SCR $(\mathbf{A})$, heart RR (B), and BP $(\mathbf{C})$. The $Y$-axes denote the area under the respective measurement curves from 5.0 seconds before a card was picked from a deck. Points are split into PCP (red lines and squares) and their HCs (green lines and circles). The bars mark standard errors. $\bullet$ Significant within-group difference, $P<0.05$; • significant between-group difference, $P<0.05$; *significant interactions between groups and card deck type, $P<0.05$.

Abbreviations: SCR, skin conductance response; RR, R wave to R wave intervals; BP, systolic blood pressure; PCP, patients with chronic pain; HCs, healthy controls. 


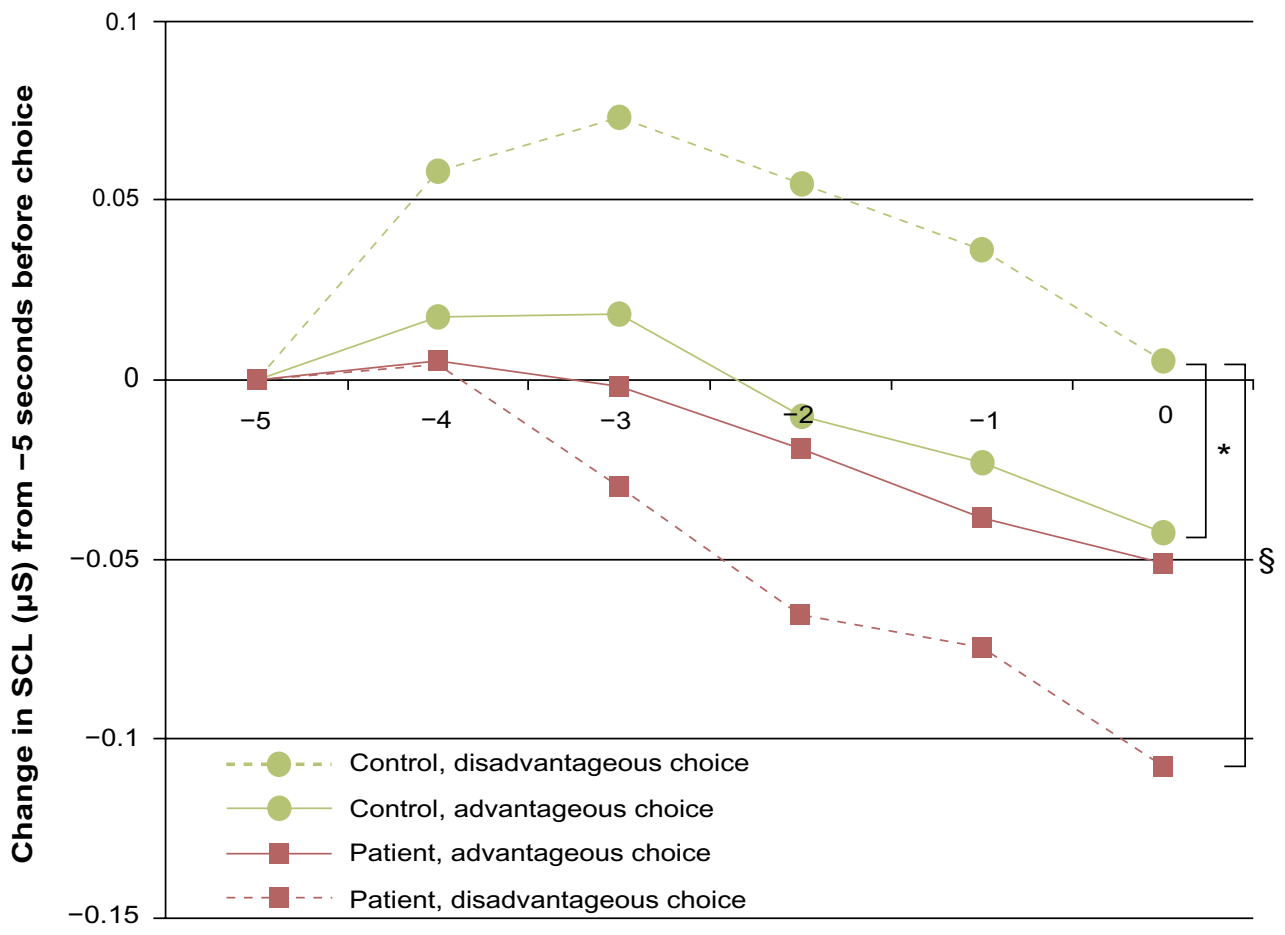

Time before choice (seconds)

Figure 5 SCR during the lowa gambling task for pain patients and controls.

Notes: The relative changes in SCL for the 5 seconds prior to making advantageous choices (continuous line) or disadvantageous choices (dashed line) for PCP (red line) and $\mathrm{HCs}$ (green line), for illustrative purposes. Curves of significantly different SCRs are marked with * or $\S$. The SCR was calculated as the area under a continuous SCL curve, with baseline as the SCL 5 seconds prior to making a choice. Simple main effects in a mixed design ANOVA showed that the SCR was significantly higher in HCs than in PCP for disadvantageous (§), but not advantageous choices. Only within the HC group was there a significant difference between the SCRs prior to making advantageous and disadvantageous choices $(*)$.

Abbreviations: SCL, skin conductance level; SCR, skin conductance response; PCP, patients with chronic pain; HCs, healthy controls; ANOVA, analysis of variance.

Table 5 SCR before disadvantageous and advantageous choices, and after receiving reward and punishment cards in the IGT in $\mathrm{PCP}$ and matched HCs

\begin{tabular}{|c|c|c|c|c|}
\hline & \multirow[t]{2}{*}{ PCP } & \multirow[t]{2}{*}{ HCs } & \multicolumn{2}{|c|}{$\begin{array}{l}\text { Between } \\
\text { groups }\end{array}$} \\
\hline & & & $P$ & d \\
\hline \multicolumn{5}{|c|}{ SCR before choice } \\
\hline Advantageous & $-0.14 \pm 0.67$ & $-0.02 \pm 0.49$ & 0.545 & 0.2 \\
\hline Disadvantageous & $-0.24 \pm 0.68$ & $0.23 \pm 0.42$ & $0.015^{*}$ & 0.86 \\
\hline \multicolumn{5}{|l|}{ Within groups } \\
\hline$P$ & 0.260 & $0.033^{*}$ & & \\
\hline$d$ & 0.16 & 0.55 & & \\
\hline \multicolumn{5}{|c|}{ SCR after choice } \\
\hline No punishment & $0.24 \pm 0.79$ & $0.08 \pm 0.50$ & 0.467 & 0.25 \\
\hline Punishment & $0.39 \pm 0.78$ & $0.03 \pm 0.73$ & 0.166 & 0.47 \\
\hline \multicolumn{5}{|l|}{ Within groups } \\
\hline$P$ & 0.224 & 0.742 & & \\
\hline$d$ & 0.19 & 0.08 & & \\
\hline
\end{tabular}

Notes: Numbers are mean values within groups \pm standard deviation in PCP with a pain self-rating of $\geq 4 / 10$ for $\geq 6$ months and in their healthy controls. Statistical differences between and within groups were explored with a two-tailed, two-sample Student's $t$-test. Significance of the Student's $t$-test is marked with $*$ for $P \leq 0.05$. Effect size is calculated as Cohen's $d$.

Abbreviations: SCR, skin conductance response; IGT, lowa gambling task; PCP, patients with chronic pain; HCs, healthy controls. four brain structures, no significant group differences were found, but a medium effect size was present for the reduced total cortical gray matter volume in the chronic pain patients (Table 2).

The chronic pain group had a significantly positive correlation between total cortical gray matter volume and IGT total score (Table 7). Moreover, there was a negative correlation between IGT total switching and amygdala volume in the chronic pain patients (Table 7). No such correlations with IGT behavior were found in the control group. All correlations remained significant after adjusting for depression levels (results not shown).

\section{Discussion}

To our knowledge, this is the first study to show that patients with chronic pain lack SCR before making disadvantageous decisions. In line with our hypothesis, the patient group was impaired at generating SCRs before choosing from disadvantageous card decks in the IGT. The other main finding in this study was that the decision-making ability in the chronic 
Table 6 Cardiac autonomic regulation during the IGT and rest in PCP and matched HCs

\begin{tabular}{|c|c|c|c|c|c|c|c|c|}
\hline & \multicolumn{2}{|l|}{ PCP } & \multicolumn{2}{|l|}{$\mathrm{HCs}$} & \multicolumn{4}{|c|}{ Between-groups tests } \\
\hline & \multirow[t]{2}{*}{ Baseline } & \multirow[t]{2}{*}{ IGT } & \multirow[t]{2}{*}{ Baseline } & \multirow[t]{2}{*}{ IGT } & \multicolumn{2}{|c|}{ Baseline } & \multicolumn{2}{|l|}{$\Delta \mathbf{I G T}$} \\
\hline & & & & & $P$ & $d$ & $P$ & $d$ \\
\hline Blood pressure & $126 \pm 18.6$ & $123 \pm 13.8$ & $125 \pm|7|$. & $123 \pm 12.7$ & 0.617 & 0.17 & 0.892 & 0.05 \\
\hline Heart rate & $73.5 \pm 8.4$ & $75.1 \pm 13.6$ & $72.4 \pm 8.1$ & $75.2 \pm 13.6$ & 0.466 & 0.25 & 0.161 & 0.48 \\
\hline Low-to-high frequency power ratio & $2.4 I \pm I .5 I$ & $2.66 \pm 3.49$ & $1.65 \pm 1.16$ & $2.55 \pm 3.39$ & 0.310 & 0.35 & 0.135 & 0.52 \\
\hline Normalized low frequency power & $62.1 \pm 16.1$ & $61 \pm 13.8$ & $54.8 \pm 14.6$ & $57.8 \pm 17.1$ & 0.583 & 0.19 & 0.294 & 0.36 \\
\hline Normalized high frequency power & $33.2 \pm 13.99$ & $35.22 \pm 12.9$ & $41.86 \pm 14.18$ & $38.46 \pm 16.4$ & 0.516 & 0.22 & 0.145 & 0.50 \\
\hline
\end{tabular}

Notes: Numbers are mean values within groups \pm standard deviation in PCP with a pain self-rating of $\geq 4 / 10$ for $\geq 6$ months and in their healthy controls. Measures are calculated for the baseline period (resting period prior to task) and during the IGT. Between-groups tests were performed on the baseline data and on the group-averaged $\Delta \mathrm{IGT}$. Statistical differences between baseline and IGT within groups were explored with a two-tailed, two-sample Student's $t$-test. Significance on the Student's $t$-test is marked with * for $P \leq 0.05$. The effect size is calculated as Cohen's $d$.

Abbreviations: IGT, lowa gambling task; PCP, patients with chronic pain; HCs, healthy controls; $\triangle \mathrm{IGT}$, increase from baseline to IGT.

pain patient group correlated positively with total cortical gray matter volume.

As predicted by the somatic marker hypothesis, a specific and significant increase in anticipatory SCR appeared when controls chose from the disadvantageous decks, and this correlated positively with the total IGT scores. This finding is in line with those from previous studies that showed a positive relationship between IGT performance and strength of the anticipatory SCR in healthy subjects. ${ }^{34-36}$ Similar findings were not present in the patient group.

The lack of the anticipatory SCRs in the patients with pain was not caused by a general impairment in SCR generation. There were no group differences in terms of the autonomic measures that indicated the presence of autonomic dysfunction in the patient group. For instance, despite abnormal anticipatory SCRs before the choices were made, the patient group exhibited similar SCRs after the choices were made as the controls when receiving punishment or reward. These results suggest that the chronic pain group was able to trigger somatic responses due to innate or learned stimuli, but they were impaired in the somatic marker structures necessary for sensing (sensory brain stem nuclei), processing (insula, somatosensory cortices, posterior cingulate cortex, and precuneus), or triggering (ventromedial prefrontal cortex, hippocampus, and dorsolateral prefrontal cortex) the somatic states during the pondering of choices (Figure 2). ${ }^{37}$ Figure 2 illustrates the possible abnormal pathways, shown as dotted lines, in the chronic pain group that could lead to the observed lack of SCR generation before making disadvantageous choices. The figure is based on the model by Bechara, ${ }^{7}$ as described in the Introduction.

Patients and controls also displayed different behavior during the IGT. The patient group switched significantly more between advantageous and disadvantageous decks compared to the controls in the performance phase of the

Table 7 Correlations between IGT behavior and autonomic measures and brain volumes in PCP and matched HCs

\begin{tabular}{|c|c|c|c|c|}
\hline & \multicolumn{2}{|l|}{ PCP } & \multicolumn{2}{|l|}{ HCs } \\
\hline & IGT score & Switching & IGT score & Switching \\
\hline \multicolumn{5}{|c|}{ Autonomic activity before IGT choices } \\
\hline SCR advantageous & 0.103 & 0.091 & 0.184 & -0.119 \\
\hline SCR disadvantageous & 0.063 & 0.203 & $0.568^{*}$ & 0.151 \\
\hline RR advantageous & 0.06 & 0.289 & 0.083 & -0.044 \\
\hline RR disadvantageous & -0.027 & 0.483 & 0.298 & 0.132 \\
\hline $\mathrm{BP}$ advantageous & -0.370 & 0.250 & 0.280 & -0.402 \\
\hline BP disadvantageous & -0.194 & 0.417 & -0.184 & -0.338 \\
\hline \multicolumn{5}{|c|}{ Combined cerebral volume in \% ICV } \\
\hline Total cortical gray matter & $0.691^{*}$ & -0.182 & 0.03 & -0.042 \\
\hline Hippocampus & 0.436 & -0.319 & 0.129 & -0.426 \\
\hline Amygdala & 0.156 & $-0.70 I^{*}$ & 0.011 & 0.061 \\
\hline Nucleus accumbens & 0.315 & -0.152 & 0.108 & -0.068 \\
\hline Brainstem & 0.068 & 0.130 & 0.099 & -0.355 \\
\hline
\end{tabular}

Notes: Numbers are Spearman's rho in PCP with a pain self-rating of $\geq 4 / 10$ for $\geq 6$ months and in their healthy controls. Cerebral volume is the combined volume of the two hemispheres in \% of ICV. Statistical differences within groups were explored with a two-tailed Spearman's rank-order correlation. Significance on Student's $t$-test is marked with * for $P \leq 0.05$.

Abbreviations: IGT, lowa gambling task; PCP, patients with chronic pain; HCs, healthy controls; SCR, skin conductance response; RR, R wave to R wave interval; BP, systolic blood pressure; ICV, intracranial volume. 
test. This is in line with results from previous studies that reported a difference in switching measures among patients. ${ }^{2,4}$ The current study did not find a significant group difference in the IGT total score between patients and controls. While this is in line with the findings from the largest study conducted thus far on the IGT in chronic pain patients, ${ }^{3}$ the opposite has been reported in two other studies. ${ }^{2,4}$ Compared with the reports on the IGT studies that exhibited group differences, ${ }^{2,4}$ our control group appears to have performed subpar, but based on a review of previous IGT studies, the HCs scored within the range of the control groups. ${ }^{38}$ Furthermore, the mean score of the current study's chronic pain group lies between the two chronic pain subgroups of the only past chronic pain study that reported mean scores of the IGT, albeit graphically. ${ }^{4}$ The current study thus suggests that increased switching and SCR deficits are more sensitive to chronic pain-induced impairments in decision making than in the total IGT score. The lack of standardized scores for the IGT and the general lack of mean score reporting complicate the interpretation and comparison between publications.

Moreover, normal IGT-scores have been seen in subjects without SCRs, ${ }^{39}$ as other body signals can help construct somatic markers in the brain. ${ }^{7}$ Thus, it is possible that other bodily signals guided the patient group's decisions. However, we failed to find any signs of increased reliance on other somatic states (ie, cardiac autonomic measures) in the patient group. Still, decision making in chronic pain patients can be supported by signaling pathways that were not monitored (for example, proprioceptive, vagal, or humoral pathways), ${ }^{7}$ or by the as-if loop between the effector structures and sensory structures that bypass the body altogether (Figure 2). Another possibility is that the chronic pain group draws on cognitive resources for decision making, as suggested by the association between cortical volumes and IGT scores in the chronic pain patients.

To our knowledge, this is the first study to show that performance on the IGT correlates with changes in the cerebral morphology of chronic pain patients. The present study demonstrated a strong positive correlation between the IGT total score and cortical gray matter volume in the patient group. Such a correlation was not found for the subcortical structures or the brain stem. Previous clinical studies have showed a relationship between IGT performance and cortical thickness of the ventromedial prefrontal cortex in patient groups with Parkinson's disease and alcoholism, ${ }^{40,41}$ but not in controls..$^{42}$ The current results could suggest that the IGT is sensitive to cortical thinning in chronic pain patients. The location of such thinning cannot be derived from the current results, but all the cortical areas involved in decision making are known to be affected in chronic pain patients. ${ }^{10}$
The present findings indicate that decision-making deficits in chronic pain patients are dependent on cortical volume rather than on reductions in subcortical structures, including the nucleus accumbens. The latter structure is suggested in the somatic marker hypothesis to be involved both in registering the somatic states and as an effector structure (Figure 2). ${ }^{37,43}$ Although this study demonstrated a reduction in nucleus accumbens volume in the patient group, which was in line with previous research, ${ }^{44}$ no correlation between the size of the nucleus accumbens and the IGT total score in the patient group was found. Furthermore, the amygdala, brainstem, and hippocampus are important for the generation of anticipatory SCRs and decision making (Figure 2). Although size alone does not determine function, their normal volume in the patient group suggests the observed anticipatory SCR impairment in this group has its neurophysiological correlates elsewhere.

Different mechanisms within the framework of the somatic marker hypothesis could explain the neurophysiology behind the altered decision making in chronic pain patients (see Figures 1 and 2). One possibility is that the processing structures (for example, the insula, cingulate cortex, or somatosensory cortices) interpret pain as part of the somatic state. Chronic pain may create a backdrop of noise that increases the time necessary for a somatic marker to form. There is some support for this speculation in the current data, as there is a weak, nonsignificant correlation between IGT score and pain rating immediately before testing (Table 2 ). A not mutually exclusive possibility is that the sensory structures or the aforementioned processing structures are affected by the abnormal amount of pain signals in chronic pain patients, making them less sensitive to normal interoceptive signals that contribute to the formation of the somatic markers. This explanation draws some support from the correlation between the IGT total score and cortical gray matter volume, assuming reduced cortical volume is indicative of reduced sensory functions.

\section{Limitations}

Unlike other decision-making tests such as the Cambridge Gambling Task, the IGT is reliant on working memory. ${ }^{45}$ Differences in cognitive abilities did not seem to contribute to the observed difference in IGT behavior between the chronic pain patients and controls since there were no correlations (data not shown) between the working memory and the different IGT scores in any of the groups.

The IGT procedure in the current study closely resembles that of the original computerized IGT, ${ }^{16}$ with a few exceptions previously described. Notably, the positions of 
all four decks were randomized on screen from subject to subject, as opposed to the original computerized IGT, where placements of the advantageous and disadvantageous decks are standardized. ${ }^{16}$ Studies have shown that decision making is affected by the placement of the options. ${ }^{17,46,47}$ Randomization of placement is a simple tool that can be used to eliminate any possible confounding effect of placement in the original IGT. ${ }^{18}$

The number of participants was relatively low, but still higher than in the other studies conducted assessing the IGT in chronic pain groups. ${ }^{2,4}$ Another limitation is the lack of a common pain etiology in the patient group. In general, the use of heterogeneous groups makes a study more vulnerable to type 2 errors, and there is indication that patients with different pain etiologies may have different changes in brain morphology. ${ }^{48}$ A recent meta-analysis of studies of changes in brain morphology in chronic pain patients did, however, find significant changes compared to controls. ${ }^{10}$ This indicates that, although pain etiology-specific differences may be found in brain morphology, different etiologies do have important common findings. The effect sizes of the reported significant findings in the current study do not indicate a type 2 error. Findings in a heterogeneous pain group have stronger external validity than do more homogeneous studies, as chronic pain patients are a mixed-etiology group in real-life settings. A finding in a mixed-etiology pain group is also less likely to be dependent on a cause underlying the pain per se, and increases the probability that the findings are related to chronic pain.

Two-tailed statistical tests were chosen due to the low number of participants in the current study to avoid a type 1 error. While this method increases the chance of a type 2 error, it ensures that any results from the current study are worth further investigation. To avoid too high a risk of a type 2 error, correction for multiple comparisons was not applied.

\section{Conclusion}

The current study shows that chronic pain patients have impaired generation of anticipatory SCRs before making disadvantageous decisions, possibly caused by the interpretation of pain as part of the somatic state, or by a reduced ability to process the somatic state due to chronic pain. It can be hypothesized that the patient group compensated for the reduction in anticipatory signals by becoming more dependent on cortical resources in their decision making, and they demonstrated increased switching between advantageous and disadvantageous decks during the IGT.
In summary, the presence of chronic pain was found to affect fundamental aspects of decision making, which may have significant implications for everyday functioning and choices in this patient group.

\section{Acknowledgment}

We would like to thank Professor Trond Sand, Department of Neurophysiology, St Olav's University Hospital, for letting us use his facilities.

\section{Disclosure}

The authors report no conflicts of interest in this work.

\section{References}

1. Landrø NI, Fors EA, Våpenstad LL, Holthe Ø, Stiles TC, Borchgrevink PC. The extent of neurocognitive dysfunction in a multidisciplinary pain centre population. Is there a relation between reported and tested neuropsychological functioning? Pain. 2013;154(7):972-977.

2. Walteros C, Sánchez-Navarro JP, Muñoz MA, Martínez-Selva JM, Chialvo D, Montoya P. Altered associative learning and emotional decision making in fibromyalgia. J Psychosom Res. 2011;70(3):294-301.

3. Verdejo-García A, López-Torrecillas F, Calandre EP, DelgadoRodríguez A, Bechara A. Executive function and decision-making in women with fibromyalgia. Arch Clin Neuropsychol. 2009;24(1): $113-122$.

4. Apkarian AV, Sosa Y, Krauss BR, et al. Chronic pain patients are impaired on an emotional decision-making task. Pain. 2004;108(1-2):129-136.

5. Bechara A, Damasio AR, Damasio H, Anderson SW. Insensitivity to future consequences following damage to human prefrontal cortex. Cognition. 1994;50(1-3):7-15.

6. Damasio AR. The somatic marker hypothesis and the possible functions of the prefrontal cortex. Philos Trans R Soc Lond B Biol Sci. 1996; 351(1346):1413-1420.

7. Reimann M, Bechara A. The somatic marker framework as a neurological theory of decision-making: review, conceptual comparisons, and future neuroeconomics research. J Econ Psychol. 2010;31(5):767-776.

8. Bechara A, Tranel D, Damasio H, Damasio AR. Failure to respond autonomically to anticipated future outcomes following damage to prefrontal cortex. Cereb Cortex. 1996;6(2):215-225.

9. Harlow JM. Recovery from the passage of an iron bar through the head. Bulletin of the Massachusetts Medical Society. 1868;2:327-347.

10. Smallwood RF, Laird AR, Ramage AE, et al. Structural brain anomalies and chronic pain: a quantitative meta-analysis of gray matter volume. J Pain. 2013;14(7):663-675.

11. Rodriguez-Raecke R, Niemeier A, Ihle K, Ruether W, May A. Structural brain changes in chronic pain reflect probably neither damage nor atrophy. PLoS One. 2013;8(2):e54475.

12. May A. Chronic pain may change the structure of the brain. Pain. 2008;137(1):7-15.

13. Williamson A, Hoggart B. Pain: a review of three commonly used pain rating scales. J Clin Nurs. 2005;14(7):798-804.

14. Apkarian AV, Baliki MN, Geha PY. Towards a theory of chronic pain. Prog Neurobiol. 2009;87(2):81-97.

15. Oldfield RC. The assessment and analysis of handedness: the Edinburgh inventory. Neuropsychologia. 1971;9(1):97-113.

16. Bechara A, Tranel D, Damasio H. Characterization of the decisionmaking deficit of patients with ventromedial prefrontal cortex lesions. Brain. 2000;123(Pt 11):2189-2202.

17. Rodway P, Schepman A, Lambert J. Preferring the one in the middle: further evidence for the centre-stage effect. Appl Cogn Psychol. 2012;26(2):215-222. 
18. Dunn BD, Dalgleish T, Lawrence AD. The somatic marker hypothesis: a critical evaluation. Neurosci Biobehav Rev. 2006;30(2): 239-271.

19. Task Force of the European Society of Cardiology and the North American Society of Pacing and Electrophysiology. Heart rate variability: standards of measurement, physiological interpretation and clinical use. Circulation. 1996;93(5):1043-1065.

20. Cleeland CS. Pain assessment in cancer. In: Osoba D, editor. Effect of Cancer on Quality of Life. Boca Raton, FL: Taylor and Francis; 1991:293-305.

21. Klepstad P, Loge JH, Borchgrevnik PC, Mendoza TR, Kaasa S. The Norwegian brief pain inventory questionnaire: translation and validation in cancer pain patients. J Pain Symptom Manage. 2002;24(5): 517-525.

22. Furman DJ, Waugh CE, Bhattacharjee K, Thompson RJ, Gotlib IH Interoceptive awareness, positive affect, and decision making in major depressive disorder. J Affect Disord. 2013;151(2):780-785.

23. Beck AT, Steer RA, Brown G. Manual for the Beck Depression Inventory-II. 2nd ed. San Antonio, TX: Psychological Corporation; 1996.

24. Bechara A, Damasio H, Tranel D, Anderson SW. Dissociation Of working memory from decision making within the human prefrontal cortex. J Neurosci. 1998;18(1):428-437.

25. Berryman C, Stanton TR, Jane Bowering K, Tabor A, McFarlane A, Lorimer Moseley G. Evidence for working memory deficits in chronic pain: a systematic review and meta-analysis. Pain. 2013;154(8): 1181-1196.

26. Fos, Lori A., Kevin W. Greve, Marne B. South, Charles Mathias, and Hope Benefield. Paced Visual Serial Addition Test: an alternative measure of information processing speed. Applied Neuropsychology. (2000);7(3):140-146.

27. Brand M, Heinze K, Labudda K, Markowitsch HJ. The role of strategies in deciding advantageously in ambiguous and risky situations. Cogn Process. 2008;9(3):159-173.

28. Cavedini, Paolo, Claudia Zorzi, Clementina Baraldi, Sara Patrini, Giuliana Salomoni, Laura Bellodi, Rafael C. Freire, and Giampaolo Perna. The somatic marker affecting decisional processes in obsessive-compulsive disorder. Cognitive Neuropsychiatry. 2012;17(2): 177-190.

29. Crone, Eveline A., and Maurits W. Van Der Molen. Development of Decision Making in School Aged Children and Adolescents: Evidence From Heart Rate and Skin Conductance Analysis. Child Development. 2007;78(4):1288-1301.

30. Lawrence, Natalia S., Sarah Wooderson, David Mataix-Cols, Rhodri David, Anne Speckens, and Mary L. Phillips. Decision making and set shifting impairments are associated with distinct symptom dimensions in obsessive-compulsive disorder. Neuropsychology. 2006;10(4): 409-419.

31. Werner, Natalie S., Stefan Duschek, and Rainer Schandry. Relationships between affective states and decision-making. International Journal of Psychophysiology. 2009;74(3):259-265.
32. Bechara A, Damasio H, Damasio AR, Lee GP. Different contributions of the human amygdala and ventromedial prefrontal cortex to decisionmaking. J Neurosci. 1999;19(13):5473-5481.

33. Brewer JB. Fully-automated volumetric MRI with normative ranges: translation to clinical practice. Behav Neurol. 2009;21(1):21-28.

34. Crone EA, Somsen RJ, Van Beek B, Van Der Molen MW. Heart rate and skin conductance analysis of antecedents and consequences of decision making. Psychophysiology. 2004;41(4):531-540.

35. Guillaume S, Jollant F, Jaussent I, Lawrence N, Malafosse A, Courtet P. Somatic markers and explicit knowledge are both involved in decisionmaking. Neuropsychologia. 2009;47(10):2120-2124.

36. Carter S, Smith Pasqualini M. Stronger autonomic response accompanies better learning: a test of Damasio's somatic marker hypothesis. Cogn Emot. 2004;18(7):901-911.

37. Bechara A. The somatic marker framework and the neurological basis of decision making. In: Ebstein R, Shamay-Tsoory S, Chew SH, editors. From DNA to Social Cognition. Hoboken, NJ: John Wiley \& Sons, Inc.; 2011:157-183.

38. Steingroever H, Wetzels R, Horstmann A, Neumann J, Wagenmakers EJ. Performance of healthy participants on the Iowa Gambling Task. Psychol Assess. 2013;25(1):180-193.

39. Heims HC, Critchley HD, Dolan R, Mathias CJ, Cipolotti L. Social and motivational functioning is not critically dependent on feedback of autonomic responses: neuropsychological evidence from patients with pure autonomic failure. Neuropsychologia. 2004;42(14):1979-1988.

40. Ibarretxe-Bilbao N, Junque C, Tolosa E, et al. Neuroanatomical correlates of impaired decision-making and facial emotion recognition in early Parkinson's disease. Eur J Neurosci. 2009;30(6):1162-1171.

41. Le Berre AP, Rauchs G, La Joie R, et al. Impaired decision-making and brain shrinkage in alcoholism. Eur Psychiatry. 2014;29(3):125-133.

42. Gansler DA, Jerram MW, Vannorsdall TD, Schretlen DJ. Hierarchical organization of cortical morphology of decision-making when deconstructing Iowa Gambling Task performance in healthy adults. $J$ Int Neuropsychol Soc. 2012;18(3):585-594.

43. Simon NW, Montgomery KS, Beas BS, et al. Dopaminergic modulation of risky decision-making. J Neurosci. 2011;31(48):17460-17470.

44. Baliki MN, Petre B, Torbey S, et al. Corticostriatal functional connectivity predicts transition to chronic back pain. Nat Neurosci. 2012;15(8):1117-1119.

45. Rogers RD, Owen AM, Middleton HC, et al. Choosing between small, likely rewards and large, unlikely rewards activates inferior and orbital prefrontal cortex. J Neurosci. 1999;19(20):9029-9038.

46. Raghubir P, Valenzuela A. Center-of-inattention: position biases in decision-making. Organ Behav Hum Decis Process. 2006;99(1): 66-80.

47. Valenzuela A, Raghubir P. Position-based beliefs: the center-stage effect. J Consum Psychol. 2009;19(2):185-196.

48. Baliki MN, Schnitzer TJ, Bauer WR, Apkarian AV. Brain morphological signatures for chronic pain. PLoS One. 2011;6(10):e26010.
Journal of Pain Research

\section{Publish your work in this journal}

The Journal of Pain Research is an international, peer-reviewed, open access, online journal that welcomes laboratory and clinical findings in the fields of pain research and the prevention and management of pain. Original research, reviews, symposium reports, hypothesis formation and commentaries are all considered for publication.

\section{Dovepress}

The manuscript management system is completely online and includes a very quick and fair peer-review system, which is all easy to use. Visit http://www.dovepress.com/testimonials.php to read real quotes from published authors. 